Voix et Images

voixetimages

\title{
Comme au commencement du langage
}

\section{Paul Chanel Malenfant}

Volume 18, numéro 2 (53), hiver 1993

Francine Noël

URI : https://id.erudit.org/iderudit/201036ar

DOI : https://doi.org/10.7202/201036ar

Aller au sommaire du numéro

\section{Éditeur(s)}

Université du Québec à Montréal

\section{ISSN}

0318-9201 (imprimé)

1705-933X (numérique)

Découvrir la revue

\section{Citer cet article}

Malenfant, P. C. (1993). Comme au commencement du langage. Voix et Images, 18(2), 404-417. https://doi.org/10.7202/201036ar d'utilisation que vous pouvez consulter en ligne.

https://apropos.erudit.org/fr/usagers/politique-dutilisation/ 


\section{Poésie}

\section{Comme au commencement du langage}

\section{Paul Chanel Malenfant, Université du Québec à Rimouski}

Les deux monosyllabes dont dispose le titre du dernier recueil de Pierre Ouellet, Fonds suivi de Faix ${ }^{1}$, participent bien de l'extrême compacité de cette poésie travaillée par le poids précis de chaque signe, par l'exacte mesure de chaque mot posé sur la page tel un spécimen du vocabulaire, chacun étant ciselé, soupesé, méticuleusement choisi et écouté à la faveur de son retentissement avec les autres. Une poétique de l'^épure - mot d'ailleurs emblématique de ce texte - s'exerce alors en ce fort, et fort beau livre, construit avec l'application et la minutie qui seraient propres à un "traité " d'existence et d'écriture: «Ma vie élaguée: par plus que vivre, où tout s'épure, / Épars, jusqu'à l'air du large, / Qui s'immisce là: entre les mots" (p. 90). Il faut écouter l'énergie acoustique discrète qui irrigue tel énoncé où "Ma vie" se concentre en l'infinitif "vivre *, où l'attache syntaxique "par plus " se raffine en le verbe "s'épure*, les deux se dilatant en cet "Épars *, qui résonne en sourdine dans "l'air du large ", lequel segment vient finalement se contenir en cette seule syllabe: "là », véritable lieu d'un élagage et d'une réduction pneumatique du sens: "entre les mots.

C'est entendre ici à la fois l'activité et la retenue d'une texture littérale qui s'emploie à endiguer la générosité d'une prolifération langagière par la pratique de la conciliation monochrome, celle que dit ce strict vers: : disséminée, et du même grain. (p. 13). Celle que désigne encore cet autre vers, justement fonctionnel, du poème inaugural intitulé "Chant donné": "- miroir où mourir sera renversé " (p. 9): le dédoublement spéculaire de ce "miroir où mourir" y active, par le calque de la lettre, une inversion et une superposition oblitératrices.

Cette compétence du poète à user des marqueurs de la poétique se reconnaît, non seulement dans la filature sonore ou le procédé anagrammatique, mais encore dans la diversité des stratégies de la syntaxe et de la typographie, dans l'adroit maniement des effets rhétoriques comme dans l'érudition des accointances intertextuelles ou culturelles. Cette adresse pourrait laisser croire à une fabrique d'écriture davantage portée par la virtuosité de l'artifice formel que par la force d'une rigoureuse introspection. Rien de tel, chez Pierre Ouellet, 
pour qui le poème se conforme toujours à une impeccable "formesens", à une stricte adéquation entre la matière et la manière textuelles. En cette poésie, il s'agit certes de saisir et de susciter le langage, de le tenir musicalement en état de veille: "Il y a des mots / Qui dorment dans d'autres / Que ma voix éveille» (p. 63). Mais cette convocation des mots qui gisent sous les mots tient à un vœu de vigilance, à une volonté de faire émerger d'eux de solides certitudes. Le poème, en sa concrétion, porte le poids d'une preuve à faire, d'un monde matériel à affirmer: "De tout chemin: jusqu'au désert / - et le fardeau de la preuve / Que ce monde existe" (p. 46).

C'est pourquoi ces poèmes s'érigent sur la page en blocs monolithiques; ils s'adonnent à la netteté tranchante de l'aphorisme ou de l'énoncé lapidaire: «On ne fait face à rien / Que ce miroir opaque /: parler aux mots / Qui ne répondent jamais / - que du mutisme où l'on se cache. (p. 56). Au silence lourd, on oppose l'écoute flottante de tous les "bruissements de la langue . À la vacuité, on fait obstacle par l'opacité d'un regard intense, insistant, un regard "pesant". Car la poésie vise à combler les vides: "En quoi même le vide / Sera repeint: bleu, avec la / Peinture à l'eau de tes yeux. (p. 22); à colmater ces irréversibles vides de l'évidence: "On sait que l'être / N'aime pas le vide / Qui le lui rend" (p. 88).

Une fois posée comme substantielle, la poésie se rend disponible à l'accueil du monde: «Le monde est clos contre quoi / Se heurte chaque mot / Dont le choc terrible dans notre voix / Fera chanter le chant plus haut: ouvert. (p. 143). Ainsi, tout un cosmos composé du ciel, des fleuves, du désert, de l'air, de ces fréquents "oiseaux" désignés comme des icônes des "mots", se déploie, sous un mode à la fois figuratif et lyrique, dans ce texte. Les éléments s'y enchevêtrent selon une rêverie du mélange et de la permutátion: "La terre s'égare dans le ciel / Où l'origine de l'orage / Brouille toute piste" (p. 114). Efficacité de ce triolet: par l'aimantation de "égare", à "origine" et à "Orage ", il crée un effet d'étalement mimétique, qui, littéralement et par effet de synesthésie, oblitère les différences sensibles. Il participe de la confusion et de l'éclosion simultanée d'une genèse. Tout se passe alors comme si la fluidité vocale qui assortit les vocables les uns aux autres tenait lieu de souffle créateur, d'une voix, à la fois révélatrice et réalisatrice, qui insuffle la vie à toutes choses.

Cette exigeante utopie de la poésie qui se déploierait comme un verbe génératif est portée ici par la fréquence des motifs de l'air et du vent, des lèvres et de la parole, de même que par les nombreuses occurrences de verbes élémentaires tels "voir ", "entendre ", "parler ": 
- Parler: rendre à l'air ce qu'on lui prend. (p. 121). Réversibilité de tout échange entre les mots et le monde: «Que le monde entier sombre: / Dans sa seule évocation. (p. 188) affirment impérieusement les derniers vers de ce livre.

Cette activité de l'ubiquité entre le réel et la parole s'inscrit dans des poèmes où l'économie lexicale même épuise les plus infimes ressources du sens, les plus fécondes capillarités de la lettre. Soit tel intense, impeccable petit morceau d'une musique sérielle:

La source se rue sur les oiseaux

Pour être bue - épuisée vive -

Et les oiseaux, dans leur chant retenu,

$Y$ vivent de la soif - leurs ailes

Sont une mer sombre et de plomb

Qui coule en elles plus leste

Que l'eau dans l'eau trop douce des ruisseaux. (p. 26)

On peut penser à une Rina Lasnier qui se serait mise à l'écoute des dispositifs du formalisme, à Eugène Guillevic pour la fascination qu'il entretient à l'endroit de tout punctum cosmique (il y a l'herbe, l'aile, la branche, la feuille, les ronces, qui sont aussi très souvent nommées, chez Pierre Ouellet), à Jacques Brault, virtuose à faire retentir le moindre timbre harmonique du langage. Pourtant, de poème en poème, dans l'effet multiplicateur et proliférant où chaque texte se trouve repris et modulé, informé par l'étendue de la musique qu'il tisse, la voix de Pierre Ouellet se démarque de tout autre "Chant donné. (p. 9). Je regrette simplement, avec d'autres lecteurs de poésie, qu'en un livre où advient avec une telle justesse «l'émotion de la forme ", où se conjoignent d'évidence le savoir de la poétique et la conscience de la poésie, se retrouvent quelques scories d'une ancienne modernité: abus des tirets, fréquent recours aux deux points en début de vers et autres petites excentricités de ponctuation ou de découpage. Pareille épure de poésie s'accommode mal, me semble$t$-il, des colifichets ou des futiles artifices du traitement de texte.

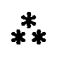

On retrouve un semblable vou d'adéquation spontanée de la pensée et de la parole dans plusieurs lieux de la poésie de François Charron, plus particulièrement sans doute dans le dépouillement presque ascétique, élémentaire, de ses derniers livres, $L a$ beauté des visages ne pèse pas sur la terre ${ }^{2}$, L'Intraduisible Amour $^{3}$ et jusqu'au plus récent, Pour les amants ${ }^{4}$. S'exprime encore, chez ce poète, une 
poétique de l'harmonie immédiate où tout désir est donné, gratuit, réalisé dans l'instant même de sa formulation: «Le ciel désire la terre, le ciel est une pierre au bout de mes doigts" (p. 9). L'usage fréquent des verbes "être", "rester " et "demeurer " de même que le recours systématique au temps de l'indicatif créent un effet de calme permanence et contribuent à glorifier la confortable qualité d'un état présent: :Être soi-même demeure intouché entre les mots" (p. 26). Le poète se plaît à évoquer l'intemporalité originelle: "Nous adhérons à l'origine en étreignant le jour qui n'a plus de nom. (p. 12); à poser l'écriture en des scènes quotidiennes de commencement et d'émergence; à vanter la disponibilité inaugurale: "Nous pouvons encore accueillir ce qui naît comme au premier jour du monde. (p. 25). En cet éloge de la lenteur réceptive, adviennent des jouissances paisibles et inédites: "Nous découvrons une lenteur utile, nos mouvements dans l'espace atteignent l'inconnu - (p. 15). Toutes pulsions se néantisent et les amants s'abolissent dans la pure transparence: "Demain, quand on se désire, est une absence d'un blanc très pur" (p. 17).

Ce défilé de phrases linéaires, sans heurts syntaxiques, finit par installer une monochromie affirmative, comme si la conscience se figeait dans sa propre béatitude et dans son ravissement: "La poésie habite l'univers. [...] L'essentiel nous est toujours donné" (p. 18). Le lecteur, même le moins sceptique ou le plus engagé dans la pensée heureuse, se trouve exclu de tant de certitudes, de tant de désinvoltes détachements: "Je sais que nous n'aurons été qu'un souvenir, qu'une attente au bout d'une allée, et que mon travail est d'oublier. (p. 21). J'ai souvent trouvé du repos, voire une réelle qualité de silence, à la lecture des récents livres, "purs et simples", de François Charron. Le propos de Pour les amants, qui glorifie la ténuité verbale, le nivellement des relations de cause à effet, la rêverie de la dilution et de l'uniformité, la satisfaction laconique à l'égard du monde, le désœuvrement sage, l'indifférence des choses, ce propos même me donne envie de bruits, de fracas, de vie fébrile.

En cette poésie de la plus simple expression, la forme semble abandonnée à son surgissement, au flottement d'une pensée vacante et laissée libre: "Je n'ai aucune idée, je reste vulnérable. La seule chose qui compte est d'être là, de recevoir la sagesse insensée du silence" (p. 70). Aucune intervention laborieuse ne vient raturer ou condenser le flux intérieur: "Je dis tout ce qui me passe par la tête. Mon écriture est fille du vent . (p. 69). Le poème se donne dans l'étale, dans le lisse, dans une pâle "présence de l'absence": "J'essaie de ne pas penser. En voulant trop saisir le pourquoi des choses, la profondeur de la personne devient une ombre. (p. 55). 
- Le rien engendre le calme. (p. 54) et *[...] il faut laisser le poème venir a soi* (p. 58): sans doute n'ai-je pas l'innocence requise pour accéder à de pareilles invitations à la vacuité ou à la passivité. Mais je ne parviens pas à habiter cet espace douillet d'une pensée blanche où ne filtrent jamais les turbulences du monde, où les amants s'enveloppent dans la grâce un peu mièvre de leur intimité. Quitte à manquer cette quête de la profondeur, dont je sais qu'elle porte, fondamentalement, toute l'œuvre de François Charron; et à reconnaître, en l'impatience de ce commentaire, qu'^Écrire est un silence que nous aurions perdu * (p. 46).

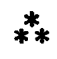

Chez Michel Pleau, dans Le corps tombe plus tard ${ }^{5}$, recueil qui a mérité en 1992 le prix Octave-Crémazie, un registre lexical référentiel qui nomme le vent, la mer, un arbre, la neige, une table et une fenêtre, suffit à créer ce climat de recueillement et de transparence intérieure souvent propre à la poésie de François Charron. Le détachement de soi, l'observation cosmique, l'évasion méditative constituent aussi, chez ce jeune poète; des états de réceptivité au monde: "personne n'écrit le poème / les amoureux sèment / le petit vent des villes / le ciel se vide d'étoiles. (p. 8). Petite pièce de concentration limpide où le découpage en mouvements brefs et la filature ludique (cette rime entre "poème " et "sèment" qui fait entendre le "s'aiment " des "amoureux", ce "petit vent des villes" placé en symétrie acoustique avec. *le ciel se viden) instaurent un progrès scénique et marquent bien l'effort d'une diction.

D'ailleurs, tout le contexte thématique de ce court mais dense recueil est porté par l'exactitude du regard: "nous regardons", "nous observons", "nous avons le regard ", "nous remarquons", ces verbes y agissent comme un véritable leitmotiv. Ce regard pénétrant accède à l'en dessous des choses et partage leurs tranquilles métamorphoses: "des fleurs poussent / au centre de la table / le silence refait surface / les mains sortent de l'eau / nos paroles sont des gestes liquides. (p. 13). Juste alchimie imaginaire de ces fleurs transmuées en les mains qui les cueillent jusqu'à ce que cette cueillaison silencieuse se diffuse, gestes, vibrations liquides, vocales... On trouve ici d'autres bonheurs d'expression matérielle qui révèlent une sensibilité très éveillée à la qualité des substances: “avec l'eau qui perd la mémoire / nous buvons l'éternité. (p. 26). La perception simultanée confère aux choses une dense concentration élémentaire, une plénitude de la sensation et 
de la jouissance: * une orange prend feu dans ma bouche / il pleut des cendres de lumière* (p. 52).

Au thème du regard qui anime les objets s'adjoint aussi celui de la marche. Les "pas", qui résonnent en maints lieux du recueil (dans "le passage blanc des passions " (p. 9) jusque "sous les pas des passants" [p. 18]), se donnent comme des décalques dynamiques des "pages", tous deux motifs métaphoriques du temps qui tourne, du temps qui passe. On trouve ainsi, placés en écho rapproché, ces deux énoncés similaires: "des pages que nous tournons / pour ne pas vieillir (p. 17) convoque le doublet, "nous courons après nos pas d'enfant / rattrapons le temps perdu à vieillir. (p. 25). Juste manière de parler du passage de l'écriture (chez un poète qui a aussi mérité le prix Alphone-Piché, en 1991, pour une suite poétique intitulée "Nous passons sous silence. [quatrième de couverture]), de cet éphémère état présent de toutes choses, cela que tente sans doute de conjurer la gravité du titre de ce recueil: Le corps tombe plus tard.

Par sa nette cohérence imaginaire, par la subtilité des dispositifs formels mis en texte dans l'espace thématique, ce livre concis place une voix très juste. Sans doute faudra-t-il réfréner quelques distorsions figuratives alambiquées comme «la nuit retourne l'univers / dans sa soupe. (p. 33), le "repère mémorique d'un visage..(p. 42) ou "les poupées de nos peurs " (p. 43). Sans doute faudra-t-il aussi procéder à une opération de rature: dans un même texte, l'alignement systématique des déterminatifs ("l'ouverture du geste ", "le passage blanc des passions", "l'ivresse des villes au matin", "la musique de nos regards", "la fenêtre brisée des souvenirs" [p. 9]) crée un effet d'inflation et de redondance. Bref, quelques notes discordantes sur une partition toute en nuances.

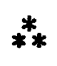

La poésie comme "réel absolu", comme idéal et destin, comme seule manière d'être au monde: tel serait le programme idéologique sans compromis dans Le Poète jeté aux chiens ${ }^{6}$, d'Yves Roy, un autre jeune auteur qui signe ici sa première œuvre. Le contrat métatextuel qui soutient ce livre de même qu'une certaine pose où se joue la dérision tonique pourraient rappeler le climat des derniers recueils d'André Roy (coïncidence onomastique, je présume), dans sa grande suite intitulée L'Accélérateur d'intensité, plus particulièrement dans le dernier volet, On sait que cela a été écrit avant et après la grande maladie?. 
- [J]'incarne un petit bonhomme de guerre / aux souffrances de style= (p. 15), écrit Yves Roy, avant de faire acte de foi dans les vertus thérapeutiques de la langue et de l'écriture: "mais, poète, celui toujours / ironisant sur ses morsures / que seule une langue peut guérir * (p. 16). L'apparente légèreté du ton, un certain égotisme dans le choix lexical, le découpage du vers à l'articulation forte, tout cela désigne, sinon une influence, à tout le moins une connivence oblique entre les deux poètes. C'est sans doute d'ailleurs dans la précision et l'efficace des opérations syntaxiques, dans la justesse des ruptures et des gonds du vers libre, que la poésie d'Yves Roy à la fois retient et laisse le sens en suspens, qu'elle parvient à créer des étonnements inédits: « Faut-il se souvenir de notre histoire / comme une lueur soudaine / dans l'été qui s'enténèbre?" (p. 28). Un vers n'attend pas l'autre quand «souvenir * et * histoire * fusionnent en * une lueur soudaine ", laquelle culmine dans l'efficace oxymoron d'un a été * qui, littéralement, “s'enténëbre ‘...

Si l'équilibre se trouve ici bien établi entre le propos et le procédé, certains autres énoncés, soutenus par une volonté de dire toute la poésie et rien que la poésie, n'accèdent pas toujours au formulaire poétique: « La poésie est manifestement / à la recherche d'un interlocuteur. (p. 34). Certes, mais on pourrait aussi écrire cela dans la section "Livres* du Devoir du samedi... Quelques dépressions laconiques ravalent ainsi ce projet d'un langage porté en marge de toutes les contingences triviales: « un poème n'est rien / qu'une bouteille lancée à la mer* (p. 22). De même le lourd retentissement d'adverbes tonitruants (*irrésistiblement ", "clandestinement ", "ultimement ") perturbe la ligne mélodique d'un texte davantage soutenu par la sourdine que par le mezzo forte. Enfin, lorsqu'il s'adonne à la méditation transcendantale, le poète n'évite pas toujours l'enflure ou la préciosité, la distorsion syntaxique créant alors un lourd effet d'éloquence: aJeté pour l'éternité, / ma chute aura été réussie. / Plus superbe est-elle encore / lorsque, subjugué par la Voix lactée, / je triomphe de Dieu / en sentant sous mon corps / la terre entière qui gît .(p. 62). Il ne vaudrait pas la peine d'insister sur ces éclats de voix, si ce n'était que ce recueil reste soulevé par une convaincante énergie, que la pensée, sollicitée tour à tour par le scepticisme ou le paradoxe, s'y illustre souvent en de justes saillies, enfin, que l'effort de structure et de contrepoint entre les parties y soit constant. Ainsi, à la difficile question que soulève la dernière section du livre, «Qu'est-ce que la poésie? », le poème terminal répond avec une juste économie de moyens: w'écris avec vous et moi. / J'écris contre vous et moi. / Voilà pourquoi ensemble / nous refermons ce livre. (p. 74). Forte manière de sceller un pacte irréversible: de la solitude de l'écriture à la solidarité de la lecture. 
Un réel engouement pour l'ambition mallarméenne d'une inscription du livre dans le livre s'exprime chez nombre de jeunes auteurs qui ne craignent pas d'affirmer d'un trait la liberté et le dur désir de la poésie. Chez Serge Patrice Thibodeau dans Le Cycle de Prague $^{8}$, de même que chez Yves Roy, le livre se donne comme espace vital; territoire fondateur de la pensée et du fait de vivre: "c'est le livre / à écrire / qui nous tient / nous tenons ouvert / le livre des choix / nous avons le choix / d'enluminer les pages / le choix d'être le livre / le choix de le fermer" (p. 38). Aveu sans parure formulé dans la saccade répétitive, dans l'insistance même d'une conviction. Et ce projet d'une adéquation identitaire au livre, voire d'une consomption en lui, est noté dans le discours de Thibodeau comme une calme exigence: "[...] ne faut-il pas simplement se faire livre soi-même, jusqu'aux cendres, jusqu'à ce qu'il ne reste plus de soi qu'un éternel moment de gratitude?» (p. 136).

Je ne résiste pas, somme toute, à l'enthousiasme de cette poésie qui se déploie tout à tour dans la concentration syncopée ou dans la prolifération lyrique, qui conjoint à la minutie de l'ascèse verbale la prodigalité imaginaire. J'avais d'ailleurs été impressionné par ces mêmes qualités de volubilité, par cette empathie spontanée à l'égard de l'esprit de tous les lieux qui s'exerçaient dans le premier livre de l'auteur, La Septième Cbute ${ }^{9}$. Disons que je les retrouve ici, à l'endroit de la ville privilégiée de Prague, mais comme en trop-plein, dans l'excédent peut-être de la surenchère ou du brio un peu facile. Je reste certes disponible à la contemplation élogieuse, à cette incantation, exaltée en leitmotiv, de tout ce qui est donné à voir: "nous fermons les yeux / immobiles, nous voyons / malgré nos paupières de pierre / nous touchons du regard / la salive et la terre mêlées" (p. 24). Je me prête volontiers aux jeux subtils de l'irradiation acoustique et sémantique: "la nuit / se taire, se terrer, la chair tarie. (p. 17). Ma lecture s'attarde à certaines scènes lumineuses installées à la manière du scintillement impressionniste: «le matin l'été / s'installe en nous / êt reste / et nous laissons luire, las / nos paupières paisibles / taillées /. dans le cristal opaque / du sommeil • (p. 69). Je condescends à l'altruisme des hommages pieux ou des tombeaux poétiques consacrés, entre autres, à "Marguerite Yourcenar" (p. 59), "Saint-Denys Garneau " (p. 53) ou *Boris Pasternak * (p. 51). Je participe enfin à l'impeccabilité de certains morceaux miniatures proches de la ciselure du haiku: "la douleur, c'est le silence / qui s'insère / entre les gouttes de pluie" (p. 76). 
Mais ces réussites nombreuses accusent davantage les circonvolutions baroques qui encombrent un texte mieux soutenu par le murmure litanique que par l'étalage d'effets bruyants. Quelque volute verbale, quelque pâmoison perturbent une poésie plus douée pour le récital que pour l'opéra: "nous, archimages / nous, caviardés par les orages / de l'ennui / à notre doigt luit une icône / un vitrage d'antéros / nous, héros sains / sobres virtuoses / aux miroirs éteints" (p. 91). Virtuose, peut-être, mais pas sobre, cet "aboli bibelot "... De même telle transe adoratrice, désireuse d'assortir la poésie à la prière et depuis longtemps inédite dans la poésie du jour, ne va pas sans naîveté: * nous sommes Un avec Dieu / et nous sommes Lumière / et le Monde en nous est immense / que portent nos prières à Dieu * (p. 46). Tout se passe comme si, à vouloir exploiter, en ses formes multiples, trop de registres mélodiques, à se poster à l'affût des leurres et des illusions du regard, la poésie de Thibodeau en venait à se confondre avec ce qu'elle observe et à «[...] s'entortiller / dans de baroques attitudes feintes* (p. 125). Ces maniérismes d'occasion, cette propension au mixage éclectique où l'éros, la mystique et l'exotisme s'interpellent, attendent encore la maturation que promettent, malgré tout, les réels lieux de profondeur et d'authenticité de cette poésie.

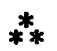

Affirmation virulente du territoire, solidarité ethnique, revendication pour la langue et l'écologie, conscience éthique exacerbée, tels sont les topoï qui portent l'engagement passionné de France Boisvert dans Massawippi ${ }^{10}$. Dans ce livre, ni murmure retenu, ni demi-teintes. La plasticité de la langue, la fougue de la nomenclature, le rythme brusque président aux messages dénonciateurs et pourraient rappeler les réquisitoires poétiques d'un Pierre Perreault ou les poèmes manifestes d'une Michèle Lalonde. Le ton est direct, abrupt, simultanément régi par la syncope et la modulation:

J'hypnotise jonquilles et narcisses

petites momies de soleils chinés

et plus tard à l'aube

dans la couche d'air jauni

l'ozone troué de biphényls polychlorés

par brassée dans une jonque sans quille

les glisserai vers la rive du lac

leurs tiges servant de mât

les larges pétales de voiles (p. 10). 
Ces «jonquilles" qui engendrent la "jonque sans quille»," comme ailleurs, ces "si lents cieux" qui se peuplent de "couleuvres" aux "mille couleurs" grouillantes (p. 11), engagent un désir de mâcher les mots en leur pulpe matérielle, comme si la diction ludique allait donner corps à toutes choses menacées d'indigence. La trituration verbale et la frénésie cumulative animent les trois suites "réalistes" du recueil, respectivement intitulées "Massawippi ", "Génération spontanée* et "Le français au secondaire ". La poésie s'y décline dans l'ardeur mnémotechnique, dans l'acharnement incantatoire:

Le discours, qui déclame ici sans ambages le mal du pays, la quête d'identité d'une "génération spontanée" et perdue à l'aube de la Révolution tranquille, les déceptions historiques et politiques, les désarrois affectifs et l'effondrement des valeurs d'une contre-culture reléguée aux oubliettes, passe par la carnavalisation verbale, par un lyrisme cassé, à l'emporte-pièce et à l'emporte-voix: "Nés entre 1960 et la crise d'Octobre / n'étions pas non plus tricot de frasques / folle équipée de bourrasques en nuées / jamais aussi hélas ah lala hallali / ou petits malins de taïga trapue / mais qui étions-nous?. (p. 20). On trouve du dépit, du courage et de l'ironie sous les éclats de voix, de la fougue juvénile par-delà la révolte et le débat polémique. Surtout, on entend sans cesse, chez France Boisvert, comme assumant tous les discords idéologiques, une véritable passion musicale pour la langue: "Peaux de pêche rose et blanche / le cœur sous le sein sous la main / or les mains dames chamanes / et les seins sous les charmes / cachés dans les soies / sous le cœur se déploie / l'amour immense pour ma langue. (p. 53). Qu'importe que cet amour condescende à quelques faciles pirouettes de poésie, celle du "verbe aisé de verts baisers " et des * cheveux fous des chevaux en bataille" (p. 22), celle "des waitresses qui stressent . (p. 25): ces jeux exorcisent peut-être "les bleus à l'âme. d'une génération qui s'est trouvée elle-même, en sa langue et en ses lieux d'appartenance, jouée.

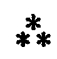

Une nostalgie du commencement du monde, de l'état brut et beau des matières premières avant que le temps n'ait déposé ses traces d'usure sur la terre, voilà ce que dit, par variantes et approfondissements successifs, toute l'œuvre de Renaud Longchamps et jusqu'au constat titulaire de son dernier recueil: Décimations: la fin des mammiferes ${ }^{11}$, lequel vient de se mériter le Grand Prix de poésie de la Fondation Les Forges 1992. Avec ce titre, qui pourrait aussi désigner 
un précis de biologie animale, le poète réaffirme l'indissociabilité des recherches scientifique et poétique et se réclame, d'une certaine manière, de la posture phénoménologique d'un Gaston Bachelard qui conjoignait le théorème et le poème. Dès lors, les vers se posent, pareils à de rigoureux syllogismes et dans la précision calculée de l'énoncé démonstratif: "Malgré la diversité / malgré l'évidence / la vie s'éteint / en nous / depuis le premier pas / depuis l'idée de la main sur la paroi" (p. 32). Nul autre artifice que cette "vie" qui s'immisce logiquement en la «diversité " et en "l'évidence ", que ce "premier pas" qui se concentre, juste écho, «sur la paroi *

Le resserrement tissulaire infléchit une intense concentration comme si l'insistance d'une texture du langage participait à la consistance même des choses: "Ce qui persiste / serait l'ombre / et son bruit / sous les pierres et les abris" (p. 11). Cette compacité littérale, qui assortit "persiste" et "pierres", "bruit " et "abris" et où "l'ombre" se trouve acoustiquement portée dans l'agglomérat *son br[...]*, marque bien une solidarité mimologique et une persistance (une résonance...) entre les mots et les choses. Chez Renaud Longchamps, l'énergie du poème, sa fibre sonore, sa juste sélection lexicale et la précision clinique des vers, traduisent l'horreur même des dissipations et des "décimations" qu'il conteste.

Il s'agit ainsi de résister à la fatigue du monde, à l'épuisement de la lumière menacée: "Je marcherai vers la lumière fatiguée / sans attendre l'univers, / futur trou noir * (p. 16). Il faut dénoncer la vieillesse prématurée de la terre soumise aux "anomalies" et aux terrorismes humains: "J'ai vu / la vieille Terre / s'effriter / dans la nuit et le vide impérial " (p. 23). Ce ton de net refus qui saisit le discours du poète devant le défaut des perspectives écologiques ( Vous avancez sur la terre / et vos yeux surveillent l'insecte, / jamais l'étoile» [p. 10]), devant les incompétences humanitaires ("Le désastre de la nature / commence / avec la faim de l'autre " [p. 40]), n'exclut jamais la fluidité d'une rêverie compassionnelle.

Ainsi, certains beaux vers, modulés par la fascination matérielle, s'adonnent-ils au flottement spéculaire de la sensation: "Mais l'eau demeure / en la mémoire de l'eau / vague" (p. 11). D'autres assistent, réfléchis et désolés, à l'innocence de toutes disparitions: "Et le rare oiseau qui s'éteint / ne sait pas lire sa chair. (p. 51); d'autres encore reconnaissent, par-delà les épuisements et les irréversibles désastres, la responsabilité généalogique: "nous sommes la chair et les os de notre temps / nous sommes les futurs ancêtres" (p. 72). L'œuvre de Renaud Longchamps, dans son intransigeant respect à l'endroit de 
toutes espèces vivantes comme dans la rare qualité de son éthique verbale, nous rappelle, de livre en livre, que "La beauté sème l'éternité / dans l'ordre du chaos" (p. 54).

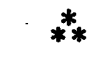

Texte énigmatique, déroutant, envoûtant aussi, que le dernier livre de Patrick Coppens, intitulé Lazare ${ }^{12}$. S'y trouve mise en scène, dans le pathos et la conscience simultanée de l'absurde, la figure emblématique de Lazare; ici dénommé Lazare Poker, tant il est vrai que dans l'ordre d'une imagination du désastre, les actions de mourir et de ressusciter ne peuvent se penser qu'en termes de spectacle et de jeu: *En comédien consommé, il se voit mourir en scène. Il laisse lentement retomber les applaudissements, un pli amer au coin supérieur de la bouche. Les bras le long du corps, il a fermé les yeux" (p. 102). Qu'on ne se trompe pas sur l'apparente dérision de la scène. Au fil d'une écriture qui brouille les cartes et les pistes, cette traversée des ombres et des lumières effectuée par le personnage allégorique de Lazare porte la métaphore même d'un itinéraire poétique.

Ponctuellement, l'instance narrative se déleste de l'anecdote référentielle pour s'adonner plutôt à la dérive exploratoire, à la liberté de la prolifération fantasmatique. L'écriture excelle alors à la ritualisation, au déploiement des étrangetés du rêve: "Lazare travaille novembre jusqu'aux entrailles de l'arène. Sa mémoire avancée sur la route de la soie tombe sous les sens d'une case de neige. La lumière a frappé l'épée et l'ombre est le fourreau de Dieu* (p. 34). Le lecteur se retrouve désormais dans le seul espace du langage, parmi "des images persévérantes ( "qu'est-ce que cela veut dire?". Il bute devant telle aberrante formule: "Dans la ville d'os, on interdit aux tapis de regarder entre les franges" (p. 30). Il se laisse happer par un paysage flou évoquant simultanément les atmosphères de Breton ou de Lautréamont: «Derrière l'invisible casino, s'étend la nuit adolescente qui prend des risques. Elle embrase ses plaintes aux minceurs insinuantes du phare. La fourche n'est pas la façon la plus naturelle de faire reculer la mer • (p. 45). Toutes les références sont abolies. Prises individuellement les choses sont reconnaissables; leur mise en perspective inédite tient de la fantasmagorie et crée l'étonnement:

En cette mort de Lazare, c'est la poésie qui participe de la vision, de la résurrection. Patrick Coppens inscrit alors des énoncés qui 
tiennent du mot de passe, de la formule magique: "À mon signal, la nacre vérifie les transparences. ( $p .53)$. Autre et juste manière de rappeler que "tout ce qui brille voit "... Il aligne des nomenclatures inédites, sortilèges verbaux ou comptines philosophiques: "Néant, zéro promu et cultivé, projet de rose et quel instant!• (p. 64). Un moment impénétrables, les appositions s'aimantent les unes aux autres, conjoignent en leur cumul le sens de l'éphémère et de l'éternité.

Le poète parvient encore à une véritable imagination onirique de la mort comme espace d'un langage bizarre, bigarré: "Pointillés et volutes, sous la paupière de l'eau qui dort, rapts et substitutions de nuages, (la scie pour la branche), imbroglio de frissons et commisérations de lèvres, impatience de viande bouillie (blanche sous l'insulte, tu serres les dents). (p. 63). Nous sommes pris au piège de ces associations venues de nulle part, emportés par le vertige de ce qui n'existe pas et pourtant existe dans l'alignement du mot à mot; pris aux mots de la mort, donc, selon le vœu même d'un poète qui manie avec brio le sarcasme, la surprise et le coup d'éclat: "Vous dites "vivre change un rêve» et je vous prends au mot" (p. 65).

\section{$\stackrel{*}{* *}$}

Je signale enfin deux grands plaisirs de poésie tout récemment posés sur ma table avec les livres de cette chronique. D'abord, l'anthologie préparée par Claude Beausoleil, Montréal est une ville de poèmes vous savez ${ }^{13}$ : l'éloquent commentaire que porte le titre tient lieu de guide en l'esprit d'un lieu, en la lettre des textes qui l'ont traversé. Ensuite, le magnifique livre d'artiste de Michel Côté, $\dot{A}$ force de silence ${ }^{14}$, publié aux Éditions du Noroît: un objet inanimé avec une âme de papier blanc. Ici, tourner les pages devient un très grand geste et le bruit que fait ce verbe sensuel, «feuilleter ", prend tout son sens.

1. Pierre Ouellet, Fonds suivi de Faix, Montréal, l'Hexagone, 1992, 192 p.

2. François Charron, La beauté des visages ne pèse pas sur la terre, Trois-Rivières, Les Écrits des Forges, 1990, 144 p.

3. Id., L'Intraduisible Amour, Trois-Rivières/Saint-Florent-des-Bois/Amay, Les Écrits des Forges/Le Dé bleu/L'Arbre à paroles, 1991, $196 \mathrm{p}$.

4. Id., Pour les amants, Montréal, Les Herbes rouges, 1992, $102 \mathrm{p}$.

5. Michel Pleau, Le corps tombe plus tard, Trois-Rivières, Les Écrits des Forges, 1992, $56 \mathrm{p}$.

6. Yves Roy, Le Poète jeté aux cbiens, Montréal, Les Herbes rouges, 1992, 80 p.

7. André Roy, On sait que cela a été écrit avant et après la grande maladie, Montréal, Les Herbes rouges, 1992, $144 \mathrm{p}$. 
8. Serge Patrice Thibodeau, Le Cycle de Prague, Moncton, Les Editions d'Acadie, $1992,160 \mathrm{p}$.

9. Id., La Septième Cbute, Moncton, Les Éditions d'Acadie, 1990, 182 p.

10. France Boisvert, Massauippi, Montréal, l'Hexagone, 1992, 66 p.

11. Renaud Longchamps, Décimations: la fin des mammiferes, Trois-Rivières, Les Ecrits des Forges, 1992, 100 p.

12. Patrick Coppens, Lazare, Montréal, Éditions Trois, 1992, 110 p.

13. Claude Beausoleil, Montréal est une ville de poèmes vous savez, Montréal, l'Hexagone, coll. . Anthologies , 1992, 316 p.

14. Michel Côté, A force de silence, Montréal, Éditions du Noroît, 1992. Non paginé. Tirage limité à 15 exemplaires. 